Pesq. Vet. Bras. 28(12):593-596, dezembro 2008

\title{
Intoxicação aguda e abortos em cobaias pelas favas de Enterolobium contortisiliquum (Leg. Mimosoideae) ${ }^{1}$
}

\author{
Josiane Bonel-Raposo 2*, Franklin Riet-Correa ${ }^{3}$, Thomas Normanton Guim², \\ Isabel Duarte Schuch ${ }^{2}$, Fabiane Boreli Grecco ${ }^{3}$ e Cristina Gevehr Fernandes ${ }^{2}$
}

\begin{abstract}
Bonel-Raposo J., Riet-Correa F., Guim T.N., Schuch I.D., Grecco F.G. \& Fernandes C.G. 2007. [Acute poisoning and abortions in guinea pigs by the pods of Enterolobium contortisiliquum (Leg. Mimosoideae).] Intoxicação aguda e abortos em cobaias pelas favas de Enterolobium contortisiliquum (Leg. Mimosoideae). Pesquisa Veterinária Brasileira 28(12):593-596. Departamento de Patologia Animal, Faculdade de Veterinária, Universidade Federal de Pelotas, Campus Universitário s/n, Pelotas, RS 96010-900, Brazil. E-mail: bonel-raposo@ brturbo.com.br

The objective was to study the acute toxicity and the abortive properties of Enterolobium contortisiliquum pods in guinea pigs. Pods of $E$. contortisiliquum were administered orally to 4 groups of 3 guinea pigs each. Another group of 3 guinea-pigs was used as control. Group 1 and 2 were fed with one dose of 5 and $10 \mathrm{~g}$ of pods for kg body weight, respectively. The guinea pigs of Group 3 and 4 received 10 and $15 \mathrm{~g} / \mathrm{kg}$, respectively, divided into daily doses of $5 \mathrm{~g} / \mathrm{kg}$. One guinea pig from Group 2 and one from Group 4 died 12 and 18 hours after the end of the administration. Gross lesions were hemorrhages of the stomach and of the large and small gut, enlarged liver, and dilated gall bladder. Histologically, the liver had severe vacuolation and necrosis of periportal hepatocytes. In another experiment a ration containing $4 \%$ of pods of $E$. contortisiliquum was fed to 2 groups of 4 guinea-pigs, 35 days after mating. Four of the 8 guinea pigs aborted 6-15 days after the beginning of ingestion. The other 4 guinea pigs were not pregnant. All guinea pigs were euthanized after abortion or at the end of the experiment. Histologically all animals had mild to severe periportal hemorrhagic necrosis. All fetuses had variable degree of autolysis. In 4 fetuses studied no significant histologic lesions were observed. The acute lesions observed in guinea-pigs are similar than those observed in the spontaneous poisoning by Enterolobium spp. in cattle. Similar lesions are observed in guinea-pigs poisoned experimentally with saponins from E. gummiferum. The results of the experiments in pregnant guinea pigs suggest that E. contortisiliquum can be used to study the abortive effect of its pods or its toxic compounds.
\end{abstract}

INDEX TERMS: Poisonous plants, hepatotoxic plants, abortion, Enterolobium contortisiliquum, Legumiosae Mimosoideae, guinea pigs.

RESUMO.- Os objetivos deste trabalho foram determinar a toxicidade aguda e o efeito abortivo de favas de Enterolobium contortisiliquum. Em um experimento frutos da árvore foram administrados a 4 grupos de 3 cobaias cada.

\footnotetext{
${ }^{1}$ Recebido em 4 de setembro de 2007.

Aceito para publicação em 26 de junho de 2008.

${ }^{2}$ Departamento de Patologia Animal, Faculdade de Veterinária, Universidade Federal de Pelotas, Campus Universitário s/n, Pelotas, RS 96010900, Brasil. *Autor para correspondência: bonel-raposo@ @ brturbo.com.br

${ }^{3}$ Hospital Veterinário, CSTR, Universidade Federal de Campina Grande, Patos, PB 58700-000. E-mail: franklin.riet@pq.cnpq.br
}

Outro grupo de 3 cobaias foi utilizado como controle. Para os Grupos 1 e 2 foram administradas 5 e $10 \mathrm{~g}$ de favas por $\mathrm{kg}$ de peso vivo, respectivamente, em doses únicas. Para os Grupos 3 e 4 foram administradas 10 e $15 \mathrm{~g} / \mathrm{kg}$, respectivamente, divididas em doses diárias de $5 \mathrm{~g} / \mathrm{kg}$. Uma cobaia do Grupo 2 e uma do Grupo 4 morreram 12 e 18 horas após o final da administração da planta. As lesões macroscópicas consistiam hemorragias no estômago e intestinos delgado e grosso, fígado aumentado e vesícula biliar distendida. Histologicamente, o fígado apresentava severa vacuolização e necrose dos hepatócitos periportais. Em outro experimento, ração contendo $4 \%$ de favas de $E$. 
contortisiliquum foi administrada a dois grupos de 4 cobaias 35 dias após o acasalamento. Das 8 cobaias, 4 abortaram 6-15 dias após o início da ingestão. As outras 4 cobaias não estavam prenhes. Duas cobaias controle pariram normalmente. Todas as cobaias foram eutanasiadas no final do experimento. Histologicamente, as cobaias que ingeriram as favas apresentaram, no fígado, necrose hemorrágica periportal de moderada a severa. Todos os fetos apresentavam graus variáveis de autólise. Em 4 fetos que foram estudados histologicamente não foram observadas lesões significantes. As lesões agudas observadas nas cobaias são semelhantes às observadas na intoxicação por favas de E. contortisiliquum em bovinos e às da intoxicação experimental com saponinas da árvore. Os resultados do experimento em cobaias prenhes demostram o efeito abortivo de E. contortisiliquum e sugerem que esta espécie pode ser utilizada para estudar o efeito abortivo da planta ou de princípios ativos identificados na mesma.

TERMOS DE INDEXAÇÃO: Plantas tóxicas, plantas hepatotóxicas, aborto, Enterolobium contortisiliquum, cobaias.

\section{INTRODUÇÃO}

Diversas species de Enterolobium são encontradas no Brasil incluindo E. contortisiliquum, E. timbouva, E. gummiferum e E. shombungkii (Lorenzi 1998). E. contortisiliquum (Vell.) Morong, conhecido como orelha de negro, orelha de macaco, timburí, timbaúba, tamboril, tambori, pau-de-sabão, timbaíba, timbó, tambaré, pacará, tamburé, é uma árvore de até $20-35 \mathrm{~m}$ de altura, encontrada en diversas regiões, desde Pará até o Rio Grande do Sul. Apresenta inflorescências branco-esverdeadas em capítulos axilares. A frutificação ocorre em junho-julho, mas as favas podem permanecer nas árvores por tempo mais longo. E. contortisiliquum se caracteriza por produzir favas pretas, com a forma de uma orelha humana, com $6-10 \mathrm{~cm}$ de comprimento (Lorenzi 1998).

No Brasil, a intoxicação por favas de E. contortisiliquum foi descrita nos Estados da Bahia (Tokarnia et al. 1999), Rio Grande do Sul, e Mato Grosso (Grecco et al. 2002) e a intoxicação por favas de E. gummiferum foi descrita en Minas Gerais (Deutsch et al. 1965). A intoxicação por E. timbouva foi descrita em São Paulo (Tokarnia et al. 1999) e Mato Grosso do Sul (Lemos et al. 1998). Os sinais clinicos da intoxicação espontânea por Enterolobium spp. são fotossensibilização (Lemos et al. 1998, Tokarnia et al. 1999, Grecco et al. 2002) e aborto (Tokarnia et al. 1999, Grecco et al. 2002). Em trabalhos de reprodução da intoxicação experimental por E. contortisiliquum e E. timbouva os principais sinais clínicos foram anorexia, depressão e diarreia, mas sem fotossensibilização (Tokarnia et al. 1960, 1999) nem aborto (Tokarnia et al. 1999). Em outros experimentos a administração de E. gummiferum (Deutsch et al. 1965) e $E$. timbouva (Lemos et al. 1998) causou fotossensibilização.

Fotossensibilização discreta foi observada por Grecco et al. (2002) em bezerros que sobreviveram a sinais digestivos agudos induzidos pela administração de favas de E. contortisiliquum. Tanto a intoxicação experimental quanto a espontânea causa degeneração e necrose hepática (Tokarnia et al. 1960, 1999, Grecco et al. 2002). Na Paraíba casos de diarréia e aborto foram constatados em caprinos que ingeriram favas de E. contortisiliquum (Benício et al. 2007).

Saponinas isoladas de E. gummiferum foram tóxicas para cobaias, mas a patologia dos animais intoxicados experimentalmente não foi estudada (Carvalho 1981). Posteriormente, essas mesmas saponinas foram administradas a cobaias, por via oral. Doses únicas de 0,5 e $1,5 \mathrm{~g} / \mathrm{kg}$ ou duas doses de $0,5 \mathrm{~g} / \mathrm{kg}$ de saponina causaram apatia, anorexia, taquipnéia, cianose, inquietação, fezes com sangue e morte em 2-8 horas após o final da administração. Na necropsia observou-se congestão e hemorragia da parede do intestino grosso que apresentava conteúdo hemorrágico. O fígado estava congesto e com aumento do padrão lobular. Na histologia observou-se severa tumefação e vacuolização fina do citoplasma dos hepatócitos, além de alguns hepatócitos apresentarem necrose. Estas lesões eram mais marcadas na região periportal, mas em alguns animais a área intermediaria $e$ centrolobulares também estavam afetadas. Os intestinos delgado e grosso estavam congestos e hemorrágicos e apresentavam necrose de células epiteliais. O coração, rim e cérebro não apresentaram lesões significativas (Carvalho et al. 2001).

Saponinas triterpénicas bismedesidicas denomindas de enterolosaponinas A e B (Mimaki et al. 2003) e contortosiliosides A, B, C, D, E e G (Mimaki et al. 2004) foram isoladas de $E$. contortisiliquum. Enterolosaponina A e contortisilioside $B$ resultaram tóxicos para macrófagos de rato e contortisilioside $A$ e $C$ resultaram tóxicas para macrófagos de rato e células de linfoma murino (Minaki et al. 2003, 2004).

Os objetivos deste trabalho foram determinar a toxicidade aguda de favas de E. contortisiliquum em cobaias e, principalmente, determinar a sua capacidade abortiva nesta espécie e desenvolver um modelo experiental para estudar as substâncias abortivas da planta.

\section{MATERIAL E MÉTODOS}

Intoxicação experimental por favas de Enterolobium contortisiliquum em cobaias (Experimento 1)

Frutos de E. contortisiliquum coletados no município de Capão do Leão foram administrados a 4 grupos de 3 cobaias cada. Outro grupo de 3 cobaias foi utilizado como controle. Das 15 cobaias utilizadas 7 eram machos e 6 fêmeas.

As cobaias foram pesadas e mantidas em jejum 24 horas antes da administração dos frutos. Estes foram triturados e diluídos em água destilada até obter-se uma consistência adequada para a administração, mas sem ultrapassar um volume máximo de $40 \mathrm{ml}$. A administração foi realizada através de sonda gástrica segundo a técnica descrita por Smith et al. (1993).

Para as cobaias dos Grupos 1 e 2 foram administradas 5 e $10 \mathrm{~g}$ de favas por $\mathrm{kg}$ de peso vivo, respectivamente, em doses únicas. Para as cobaias dos Grupos 3 e 4 foram administradas 10 e $15 \mathrm{~g} / \mathrm{kg}$, respectivamente, divididas em doses diárias de $5 \mathrm{~g} / \mathrm{kg}$. O Grupo 5 foi utilizado como controle. Dois animais que morreram 
em conseqüência da intoxicação e um do grupo controle foram necropsiados, coletando-se fragmentos de coração, pulmão, rins, baço, fígado, estômago, intestinos e sistema nervoso central que foram fixados em formol tamponado a $10 \%$, incluídos em parafina, cortados em secções de $5 \mu \mathrm{m}$ e corados por hematoxilina-eosina (HE) para estudo histopatológico.

Toxicidade de Enterolobium contortisiliquum em cobaias prenhes (Experimento 2)

As favas de E. contortisiliquum utilizados para reprodução experimental, foram coletadas no município de Capão do Leão. Estas favas foram secas à sombra, trituradas e misturadas em ração comercial para cobaias previamente moída. A ração contendo $4 \%$ de favas foi misturada com $10 \%$ de uma solução preparada com 1 litro de água fervendo e $200 \mathrm{~g}$ de amido de milho, produzindo-se a partir dessa massa homogenizada os pelets que, posteriormente, foram secados a temperatura de $37^{\circ} \mathrm{C}$ em estufa.

A determinação do dia zero (0) de prenhez das cobaias foi realizada mediante de lavagem vaginal com solução salina, onde se procurava a presença de espermatozóides.

Dois grupos de 5 cobaias foram acasaladas com um macho cada. Inicialmente os machos foram deixados separados das fêmeas em caixas vizinhas por uma semana. Após esse período foram mescladas com o macho e realizados lavados vaginais diários nas fêmeas. O dia em que foram observados espermatozóides no lavado vaginal foi considerado o dia 0 de gestação.

Dois grupos de 4 cobaias cada receberam os pelets contendo $4 \%$ de favas de E. contortisiliquum a partir do $35^{\circ}$ dia de gestação (considerado terço final da gestação), na dose de $35 \mathrm{~g}$ diárias, que é o consumo normal de ração de uma cobaia normal ao dia. O Grupo 1 recebeu a ração experimental por 10 dias e o Grupo 2 por 15 dias. Cada grupo teve um animal como controle que recebeu ração comercial nas mesmas proporções. Diariamente as cobaias foram avaliadas clinicamente. Após a constatação de aborto ou parto, houve suspensão da administração dos pelets e as cobaias foram eutanasiadas com $0,3 \mathrm{ml}$ de tiopental, via intra tecal, após sedação inicial com eter etílico, conforme critérios que prevê a resolução do Conselho Regional Medicina Veterinária. As cobaias que não abortaram ou pariram durante o período experimental foram eutanasiadas ao final do mesmo constatando-se se estavam prenhes $A$ prenhez em cobaias dura em média 59 dias podendo chegar até 67 dias. Na necropsia foram coletados todo o aparelho genital e fragmentos de fígado, que foram fixados em formol tamponado a $10 \%$, e processado conforme já descrito acima.

\section{RESULTADOS \\ Intoxicação experimental por favas de Enterolobium contortisiliquum em cobaias (Experimento 1)}

As cobaias de todos os grupos que receberam a planta apresentaram perda de peso. Uma cobaia do Gupo 2 e outra do Gupo 4, que receberam $10 \mathrm{~g} / \mathrm{kg}$ em dose única e $15 \mathrm{~g} / \mathrm{kg}$ em três doses diárias, respectivamente, apresentaram prostração e morreram 12 e 18 horas após o final da administração da planta. Os demais animais se recuperaram espontaneamente. As lesões macroscópicas consistiam de áreas hemorrágicas, na camada serosa e mucosa do estômago, intestino delgado e grosso (Fig.1). Em ambos os animais o fígado estava levemente aumentado e a vesícula biliar distendida. As lesões histológicas caracterizaram-se por hemorragia e congestão do baço,

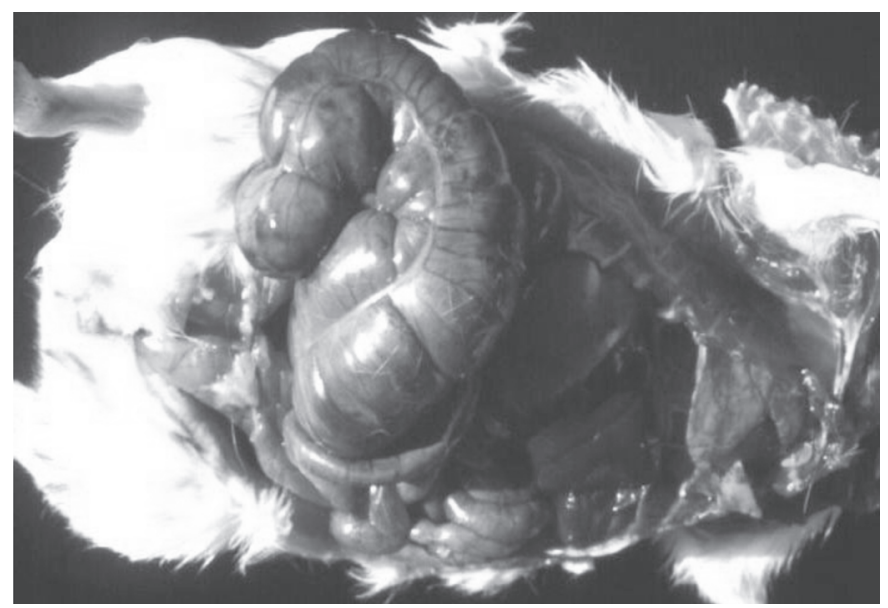

Fig.1. Cobaia intoxicada por com $10 \mathrm{~g} / \mathrm{kg}$ de favas de Enterolobium contortisiliquum. Observam-se severas hemorragias na parede do intestino grosso.

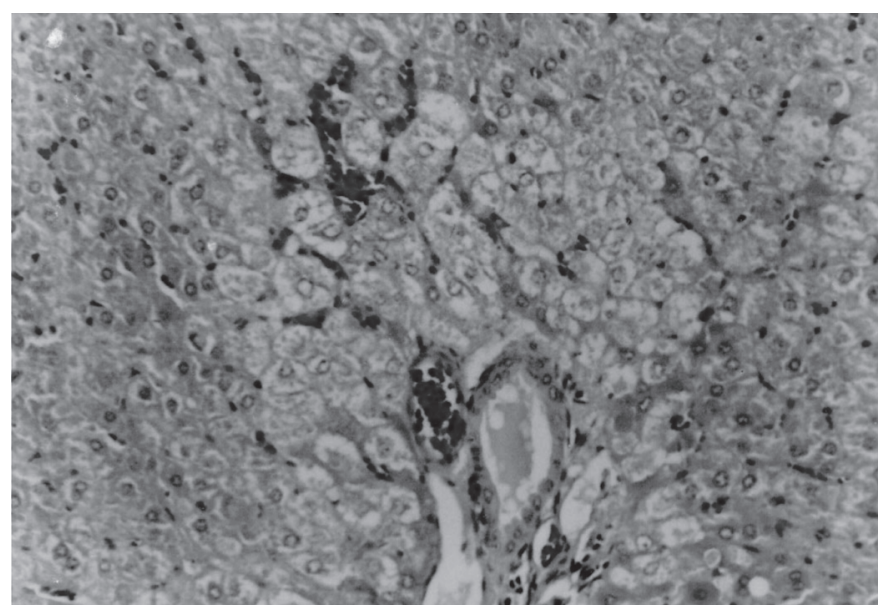

Fig.2. Fígado de cobaia intoxicada com $10 \mathrm{~g} / \mathrm{kg}$ de favas de Enterolobium contortisiliquum. Há severa vacuolização e necrose dos hepatócitos periportais. HE, obj.20x.

rins e intestino incluindo as placas de Payer. Neste último, podia ser observada discreta vacuolização das células epiteliais de alguns túbulos renais. O fígado apresentava severa vacuolização e necrose dos hepatócitos periportais (Fig.2). A cobaia do grupo controle, que foi eutanasiada, apresentou degeneração gordurosa de hepatócitos da área centrolobular. Os demais órgãos coletados não apresentaram lesões significativas.

\section{Toxicidade de Enterolobium contortisiliquum em co- baias prenhes (Experimento 2)}

Uma cobaia do Grupo 1 abortou 3 fetos (Fig.3), 8 dias após o início da ingestão da ração contendo favas de $E$. contortisiliquum. As outras 3 cobaias ingeriram a ração experimental por 10 dias, sem apresentar sinais clínicos. Após a eutanásia destes animais, 11 dias após inicio do experimento, foi constatado que nenhuma estava prenhe. A cobaia controle do grupo pariu normalmente 4 filhotes. Das 4 cobaias do Grupo 2, duas abortaram 3 fetos, nos 


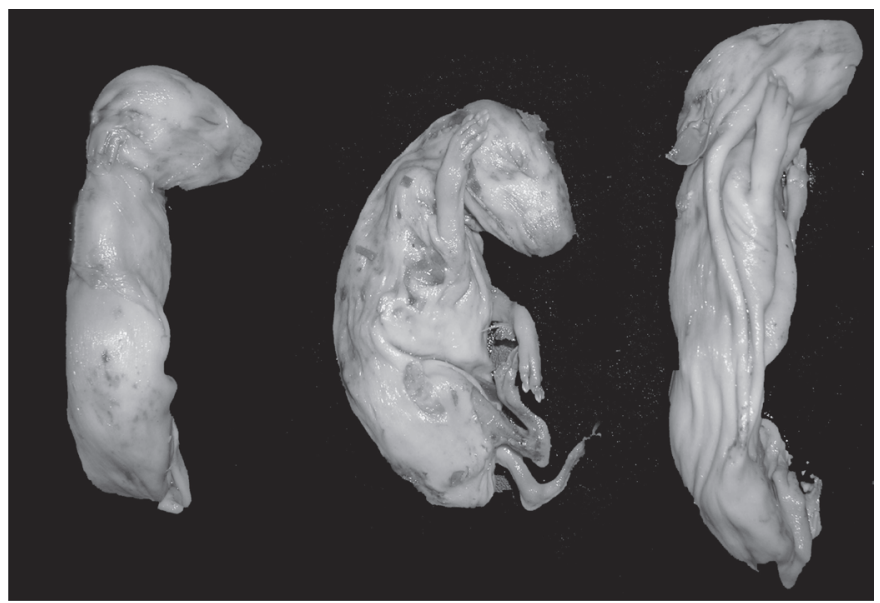

Fig.3. Fetos abortados da cobaia que abortou após ter ingerido ração contendo $4 \%$ de favas de Enterolobium contortisiliquum durante 8 dias

dias 6 e 7 após o início da ingestão. Outra abortou 5 fetos no $15^{\circ}$ dia após o início da ingestão. Na outra cobaia, que ingeriu a ração contendo favas de $E$. contortisiliquum durante 15 dias, foi constatado, na necropsia, realizada no $16^{\circ}$ dia, que não estava prenhe. A cobaia testemunha deste grupo pariu normalmente 3 filhotes.

No estudo histopatológico, as principais alterações hepáticas observadas em todas as fêmeas que ingeriram ração com favas de E. contortisiliquum foram de necrose hemorrágica periportal de moderada a severa, além de congestão dos sinusóides. Nos órgãos do sistema reprodutor não se observaram alterações significativas além daquelas que ocorrem com freqüência nos abortos independentes de sua etiologia, quais sejam: espessamento endometrial e secreção mucosa na luz endometrial e das trompas uterinas, congestão dos vasos uterinos e regressão de corpo lúteo ovariano. Todos os fetos apresentavam graus variáveis de autólise. Em 4 fetos que foram estudados histologicamente não foram observadas lesões significantes.

\section{DISCUSSÃO}

Em bovinos a intoxicação por Enterolobium contortisiliquum caracteriza-se por alterações do sistema digestivo, fotossensibilização, lesões hepáticas e abortos. Nos experimentos de intoxicação aguda em cobaias as lesões foram localizadas, também, no fígado e intestinos demonstrando que esta espécie pode ser utilizada como animal experimental para estudar o princípio ativo de Enterolobium spp. As lesões agudas observadas nos cobaias experimentais são semelhantes às observadas na intoxicação experimental com saponinas de E.gummiferum (Carvalho et al. 2001) o que sugere que estas lesões sejam causadas pelas saponinas contidas nas favas de $E$. contortosiliquum (Minaki et al. 2003, 2004).

No Experimento 2, a indução de abortos em todas as cobaias que estavam prenhes demostra a capacidade abortiva da planta. Abortos são freqüentes em vacas pre- nhes intoxicadas por E. contortisiliquum (Tokarnia et al. 1999, Grecco et al. 2002), mas não têm sido reproduzidos experimentalmente em vacas prenhes (Tokarnia et al. 1999). Apesar de que todas as cobaias apresentaram algum grau de lesão hepática não é possivel determinar se os abortos foram causados em conseqüência dessas lesões ou por um efeito direto das saponinas ou outras substâncias contidas na planta sobre o sistema reprodutor das cobaias ou causando a morte dos fetos.

Os resultados do Experimento 2 demostram que cobaias prenhes poderão ser utilizadas para estudar o efeito abortivo das favas de Enterolobium spp., mediante a administração de favas ou de substâncias identificadas nas mesmas. Também poderá ser utilizada esta espécie para o estudo de outras plantas abortivas do Brasil como Ateleia glazioviana, Tetrapterys spp., Aspidosperma pyrifolium e Stryphnodendron spp.

\section{REFERÊNCIAS}

Benício T.M.A., Nardelli M.J., Nogueira F.R.B., Araújo J.A.S. \& RietCorrea F. 2007. Intoxication by the pods of Enterolobium contortisiliquum in goats, p.80-85. In: Panter K.E., Wierenga T.L. \& Pfister J.A. (Ed.), Poisonous Plants: global research and solutions. CABI Publishing, Wallingford, Oxon, UK.

Carvalho L.R. 1981. Estudos químico e biológico de uma saponina de Enterolobium gummiferum. Dissertação de Mestrado, USP, São Paulo. 83p.

Carvalho L.R., Haraguchi M. \& Riet-Correa F. 2001. Dados não publicados (Centro de Saúde e Tecnologia Rural, Universidade Federal de Campina Grande, Patos, Paraíba).

Deutsch J., Döbereiner J. \& Tokarnia C.H. 1965. Fotossensibilidade hepatogênica em bovinos na intoxicação pela fava de Enterolobium gummiferum. Anais IX Congr. Int. Pastagens, São Paulo, p.1279-1282.

Grecco F.B., Dantas A.F.M., Riet-Correa F., Leite C.G.D. \& Raposo J.B. 2002. Cattle intoxication from Enterolobium contortisiliquum pods. Vet. Human Toxicol. 44(3):160-162.

Lemos R.A., Purisco L., Nakazato L. \& Dutra I.S. 1998. Intoxicação por Enterolobium contortisiliquum, p.307-312. In: Lemos R.A.A. (Ed.), Principais Enfermidades de Bovinos de Corte do Mato Grosso do Sul. Universidade Federal do Mato Grosso do Sul, Campo Grande, MS.

Lorenzi H. 1998. Árvores Brasileiras: Manual de Identificação e Cultivo de Plantas Arbóreas do Brasil. Instituto Plantarum de Estudos da Flora Ltda. $2^{\underline{a}}$ ed. Editora Plantarum, Nova Odessa, São Paulo.

Mimaki Y., Harada H., Sakuma C., Haraguchi M., Yui S., Kudo T., Yamazaki M. \& Sashida Y. 2003. Enterolosaponins A and B, novel triterpene bisdesmosides from Enterolobium contortisiliquum, and evaluation for their macrophage-oriented cytotoxic activity. Bioorganic and Medicinal Chemistry Letters 13:623-627.

Mimaki Y., Harada H., Sakuma C., Haraguchi M., Yui S., Kudo T., Yamazaki M. \& Sashida Y. 2004. Contortisiliosides A-G: isolation of seven new triterpene bisdesmosides from Enterolobium contortisiliquum and their cytotoxic activity. Helvetica Chimica Acta 87:851-865.

Smith B.L., Flâoyen A. \& Embling P.P. 1993. A simple gag for the intragastric dosing of guinea-pigs (Cavia porcellus). Laboratory Animals 27:286-288.

Tokarnia C.H., Canella C.F.C. \& Döbereiner J. 1960. Intoxicação experimental pela fava da "Timbaúba', Enterolobium contortisiliquum (Vell) Morong, em Bovinos. Arqs Inst. Biol. Animal, Rio de J., 3:73-381.

Tokarnia C.H., Döbereiner J., Dutra I.S., Brito I.S., Chagas B.R., França T.N. \& Brust L.A.G. 1999. Experimentos em bovinos com favas de Enterolobium contortisiliquum e Enterolobium timbouva para verificar propriedades fotossensibilizantes e/ou abortivas. Pesq. Vet. Bras. 19(1):39-45. 